\title{
Mechanisms and Treatment of Blast Induced Hearing Loss
}

\author{
Chul-Hee Choi \\ Department of Audiology and Speech-Language Pathology, Research Institute of Biomimetic Sensory Control, and \\ Catholic Hearing Voice Speech Center, Catholic University of Daegu, Gyeongsan, Korea
}

Received November 11, 2012

Revised November 11, 2012

Accepted November 15, 2012

\section{Address for correspondence Chul-Hee Choi, $\mathrm{PhD}$ \\ Department of Audiology and Speech-Language Pathology, Research Institute of Biomimetic Sensory Control, and Catholic Hearing Voice Speech Center, Catholic University of Daegu, 13-13 Hayang-ro, Hayang-eup, Gyeongsan 712-702, Korea \\ Tel +82-53-850-2541 \\ Fax +82-53-850-3383 \\ E-mail_choi@cu.ac.kr}

\begin{abstract}
The main objective of this study is to provide an overview of the basic mechanisms of blast induced hearing loss and review pharmacological treatments or interventions that can reduce or inhibit blast induced hearing loss. The mechanisms of blast induced hearing loss have been studied in experimental animal models mimicking features of damage or injury seen in human. Blast induced hearing loss is characterized by perforation and rupture of the tympanic membrane, ossicular damage, basilar membrane damage, inner and outer hair cell loss, rupture of round window, changes in chemical components of cochlear fluid, vasospasm, ischemia, oxidative stress, excitotoxicity, hematoma, and hemorrhage in both animals and humans. These histopathological consequences of blast exposure can induce hearing loss, tinnitus, dizziness, and headache. The pharmacological approaches to block or inhibit some of the auditory pathological consequences caused by blast exposure have been developed with antioxidant drugs such as 2,4-disulfonyl $\alpha$-phenyl tertiary butyl nitrone (HXY-059, now called HPN-07) and $\mathrm{N}$-acetylcysteine (NAC). A combination of antioxidant drugs (HPN-07 and NAC) was administered to reduce blast induced cochlear damage and hearing loss. The combination of the antioxidant drugs can prevent or treat blast induced hearing loss by reducing damage to the mechanical and neural component of the auditory system. Although information of the underlying mechanisms and treatment of blast induced hearing loss are provided, further and deep research should be achieved due to the limited and controversial knowledge.
\end{abstract}

Korean J Audiol 2012;16:103-107

KEY WORDS: Blast · Hearing loss · Blast injury · Blast induced hearing loss · Antioxidant Oxidative stress $\cdot \mathrm{HPN}-07 \cdot \mathrm{NAC}$.

\section{Introduction}

Noise induced hearing loss is one of the most common causes of hearing loss. Noise induced hearing loss includes a short exposure to an intense impulse noise from 100 to 150 $\mathrm{dB}$ sound pressure level (SPL), called an acute acoustic trauma and a continuous exposure to loud sounds at or above 85 $\mathrm{dB}$ SPL for relatively long period of time, called chronic noise induced hearing loss at military environments and working places. ${ }^{1-4)}$ In category of noise induced hearing loss, blast induced hearing loss seems to be included in the acute acoustic trauma because of the arbitrary distinction between impulse noise and blast. Although both impulse noise and blast can be classified in terms of the source of the pressure or the characteristics of their waveforms, blast should be differentiated from impulse noise based on the following criteria: 1) peak overpressures of blast generate tens of $\mathrm{kPa}$ whereas those of impulse noise usually less than $160 \mathrm{~dB}(2 \mathrm{kPa}), 2)$ blast produces considerable movement of air and combustion products whereas impulse noise does not, and 3) impulse noise is often related with low frequency mechanical clatter. ${ }^{5,6}$

Blast refers to a high intensity disturbance in the ambient air pressure, called blast overpressure when solids or liquids are rapidly converted into gas. ${ }^{7,8)}$ Usually, in the military, blast overpressure occurs in two situations: muzzle blast from firing heavy weapons and the detonation of explosives and munitions as in incoming artillery rounds. ${ }^{8)}$ In addition to the military environments, civilian exposures to blast overpressure have been increased in recent years due to increases in urban terrorist activities and common availability of gun propellant or high-charge explosive in most countries. ${ }^{9}$

Experimental studies of blast injury in animal models have proven that the auditory system is one of the most vulnerable systems associated with blast overpressure. ${ }^{10)}$ It has been clin- 
ically reported that permanent sensorineural hearing loss rates associated with blast overpressure in military personnel range from 33 to $78 \%{ }^{11)}$ Although there is high prevalence in hearing loss associated with blast overpressure, the mechanisms of blast induced hearing loss have not been well understood. Therefore, the objective of this study is to provide an overview of the basic mechanisms of blast induced hearing loss and review pharmacological treatments or interventions that reduce or inhibit blast induced hearing loss. In addition, this study will provide a clear analysis and understanding of research direction and possible treatment of blast induced hearing loss in future.

\section{Characteristics of Blast Waves}

When a solid or liquid explosive material is rapidly converted into a gas, blast is generated by instantaneously increased pressure of air. ${ }^{7,12,13)}$ The compression of air expands rapidly into the surrounding air at supersonic speeds and produces a heating. This induces a very sharp increase in the surrounding atmospheric pressure, known as a shock wave. The shock wave refers to a maximal or peak pressure immediately after the explosion. After the initial maximal pressure, the atmospheric pressure drops rapidly with the distance, known as underpressure wave, producing a superheated blast wind. ${ }^{7}$ The drop in pressure is represented by a negative wave of low amplitude lasting 10 times as long as the shock wave. ${ }^{13)}$ This drop is followed by a second positive pressure before atmospheric pressure returns to normal. ${ }^{12)}$ Characteristics of the blast wave can be determined by a pressure time history representing the amount of pressure as a function of time. There are three different types of the blast wave in terms of the pressure time history. ${ }^{14)}$ The typical type of the blast wave is called the Friedlander wave, a classical wave with a positive phase above atmospheric pressure and negative phase below atmospheric pressure. The second type is a simple free field wave which has a predominantly positive and negative phase with other peaks and troughs resulting from artifact, vibration, or one reflective surface. The third type is a complex wave with multiple positive and negative phases representing the initial pressure wave followed by a series of secondary wave. The complex wave may result from the initial wave reflecting off surfaces (room, bunker, foxhole, structure with a roof only) or from large shoulder-fired weapons. However, when the blast occurs in a liquid medium (water), there is no negative pressure phase. ${ }^{13)}$ Therefore, it is important to note that the types of the blast wave are decided by where the blast occurs: outdoors, within an enclosure, or water.

\section{Classification of Blast Injuries}

Blast overpressure may cause various damages and injuries of the human body. The effects of blast on human body are determined $\mathrm{b}$ a variety of factors: distance from the blast, orientation towards the blast, environmental features resulting in reflection and resonance of the blast wave, power of the blast itself, and patient's previous trauma history. ${ }^{11)}$

Blast injuries can be classified into four different categories: primary, secondary, tertiary, quaternary, and quinary injuries. ${ }^{7,912,14,15)}$ Primary blast injuries represent damages or injuries resulting from the overpressure or underpressure wave itself. Primary blast injury affects air-filled or fluid-filled organs and structures within the body such as brain, ear, lung, blood vessels, and the gastrointestinal tract. ${ }^{7,15)}$ Secondary blast injuries refer to damages or injuries resulting from flying debris or shrapnel like bomb fragments and can affect any part of the body. ${ }^{7,15)}$ Tertiary blast injuries refer to damages or injuries caused by the blast wind movement such as fractures, blunt trauma injury, and amputations. ${ }^{7,15)}$ Quaternary blast injuries include burns, inhalation of toxic fumes, crush injuries, and exacerbation of chronic diseases like asthma and psychiatric disorders. ${ }^{715)}$ Quinary blast injuries indicate a constant hyperinflammatory state syndrome caused by the toxic substances in the blast explosive being inhaled or absorbed through skin like penta-erythritol-tetra-nitrate. Quinary blast injuries occurs after attack with incendiary devices. ${ }^{15)}$

\section{Mechanism of Blast Induced Hearing Loss}

As we discussed above, damages or injuries of the auditory system are typically considered one of the primary blast injuries. Generally, the blast overpressure can induce all types of hearing loss: conductive, sensorineural, and mixed hearing loss. Clinical studies of blast injury on the auditory system have reported that approximately $60 \%$ of the patients evaluated have any types of hearing loss. ${ }^{7)}$ The degree of hearing loss ranged from mild to profound hearing loss. ${ }^{16)}$ In the outer ear, flying debris of the blast can induce damages or injuries of the pinna and the external ear canal. In the middle ear, the blast can produce damages from injection of blood vessels, subepithelial hemorrhages, small split-like perforations to multiple or total perforations. ${ }^{5)}$ The prevalence of the tympanic membrane perforation was about $32 \%$ of patients who were evaluated. ${ }^{7)}$ The frequency of perforation varied from as low as $16 \%$ to as high as $29 \%{ }^{11)}$ The perforations of the tympanic membrane were found $60 \%$ at central, $25 \%$ at anterior, and $15 \%$ at postero-superior. ${ }^{17)}$ The blast can also induce raptures 
of the tympanic membrane. Although the earliest experiments of the blast overpressure on the human ear reported that the lowest pressure of the tympanic membrane rapture is $37 \mathrm{kPa}$ (5.4 psi), there were considerable variations between individuals in the susceptibility of a tympanic membrane to rapture. ${ }^{5)}$ The raptures of the tympanic membrane can make small fragments of keratinizing squamous epithelium from the outer surface of the tympanic membrane throughout the middle ear and mastoid system, which result in masses of destructive keratinous debris within the ear, called cholesteatoma. ${ }^{18)}$ In addition, the blast can evoke other middle ear damages such as disarticulation and fracture of the ossicular chains. ${ }^{5-7,19,20)}$ The ossicular damages range from no cases to $34 \%$ of the ears undergoing tympanoplasty including incudomalleolar joint disruption (25\%), incudo-stapedial joint disruption (4\%), incudo-stapedial disruption and stapes superstructure fracture (2\%), incus dislocation $(2 \%)$, and fractured stapes superstructure $(1 \%){ }^{21)}$ The fracture or dislocation of the stapes footplate was also induced by the blast. ${ }^{22)}$

The damages caused by blast are observed within the cochlea. The cochlea is an organ that is most vulnerable to the blast overpressure. Within the cochlea, the basilar membrane was mechanically damaged by the extreme force exerted on the basilar membrane, which can rupture the inner and outer hair cells from the supporting cell attachments on the basilar membrane. ${ }^{8)}$ This may result in changes in the integrity of the tight cell junctions of the reticular lamina, changes in the membrane permeability, or holes in the reticular lamina, which allow mixing of the perilymph and endolymph causing the alterations of the ionic environment of the cochlear fluids. ${ }^{5)}$ Loss of hair cells was also induced by blast. However, there were considerable differences in the amount of damage between the outer hair cells and inner hair cells. The outer hair cells were more damaged than the inner hair cells. ${ }^{502}$ The main morphological changes observed in the outer hair cells were bleb formation, disarrangement, swelling, fusion, and break off or disappearance. ${ }^{20)}$ These damages to hair cells were more marked in the basal turns than in apical turns. A perilymph fistula from a rupture in the round window was observed. ${ }^{6)}$ In addition, when blast exposure was prolonged to $1 \mathrm{~s}$, the damage to the hair cells became more severe. ${ }^{20)}$

There is considerable variability in the effects of blast exposure on the auditory system within several species (chinchilla, pigs, and sheep). Although there is a similarity among different species in the damage pattern of the organ of Corti, the chinchilla was most susceptible to the middle ear injury, followed by the sheep and then the pigs, but the sheep was more susceptible to cochlear damage than the pig. ${ }^{19)}$

In addition to hearing loss, blast exposure may induce tin- nitus, dizziness, and headache. The probability of tinnitus resulting from the blast exposure ranged from 9 to $19 \%{ }^{7)}$ Based on the prevalence of patient-reported otologic complaints caused by the blast, tinnitus and dizziness were $49 \%$ and $15 \%$, respectively while others (headache and pain) was $30 \%$. $^{7)}$ The exposure to a single $10 \mathrm{msec}$ blast at 14 psi or $194 \mathrm{~dB}$ SPL in rats induced early onset of tinnitus and central hearing impairment at a broad frequency range. ${ }^{5,23)}$ There are significant damages of the inferior colliculus and medial geniculate body but no changes in the corpus callosum. This indicates that these damages may mainly result from the auditory pathways rather than from direct impact onto the brain parenchyma. ${ }^{23)}$ Histopathological consequences of the inner ear caused by blast overpressure include hematoma and hemorrhage within the perilymph. ${ }^{11)}$ This indicates that subarachnoid hemorrhage is a major contributor to the blast related dizziness and headache.

On the other hand, many experimental studies have reported that blast overpressure can induce damages and injuries of the brain. Direct injuries to brain may result from secondary and tertiary blast injuries. The effects of primary blast injury on the brain are not clear. The probability of brain injury induced by the blast exposure ranged from 10 to $20 \%$ of veterans returning from the wars in Iraq and Afghanistan. ${ }^{12)}$ There are specific patterns of blast induced brain injury. The brain injury occurs through elevations in cerebrospinal fluid or venous pressure due to compression of the thorax and abdomen. ${ }^{24)}$ Additional injury may result from carbon monoxide or other gas inhalation. Brain injuries are usually accompanied by injuries of other organs such as lung, bones, gastrointestinal tract, and heart, which can produce air emboli, fat emboli, and hypoxia/ischemia. ${ }^{1424)}$ The effects of blast injuries on the brain of animals include changes in small, unmyelinated axons that are characteristic of diffuse axonal injury, skull fractures, and extensive epidural, subdural, subarachnoid, and intracerebral hemorrhages, expanded perineuronal spaces, appearance of cytoplasmic vacuoles, myelin deformation, axoplasmic shrinkage, transient alternations in the distribution of neurofilament proteins. ${ }^{12,24,25)}$ These changes such as expanded perineuronal spaces, appearance of cytoplasmic vacuoles, myelin deformation, axoplasmic shrinkage are due to the activation of inducible nitric oxide synthase and oxidative stress in the brain. ${ }^{12)}$ The effects of blast injuries on the brain of humans include small or microscopic intracerebral and leptomeningeal hemorrhages and bleeds. ${ }^{24)}$ Hemorrhages occur in subcortical white matter, corpus callosum, and basal ganglia, which may result from carbon monoxide intoxication and air and fat emboli. Bleeding cases include cerebral edema, petechial and meningeal hemorrhages, cerebral 
contusion and laceration, intracerebral and subdural hematoma, and epidural hematoma. ${ }^{24,26)}$

\section{Treatment of Blast Induced Hearing Loss}

There are no direct evidences of increased oxidative stress in the auditory system induced by blast exposure but both steady state noise induced and impulse noise induced hearing loss are directly related with increases in oxidative stress after noise exposure. Oxidative stress is a severe disturbance in the balance between production of reactive oxygen species (ROS) and the antioxidant defenses. ${ }^{27)}$ Antioxidant drugs can be administered to restore the balance between production of ROS and the antioxidant defenses. ${ }^{1-3,10,28-30)}$ The pharmacological approaches to block some of the non-auditory pathological consequences caused by blast exposure have been developed with several antioxidant drugs. N-acetylcysteine (NAC) amide was effective to reduce the inflammatory response and infiltration of neutrophils in the lungs of chinchillas. ${ }^{31)}$ NAC was efficient to restore mitochondrial electron transfer, energy coupling capacity, calcium uptake activity, and reduced calcium content absorbed to brain mitochondrial membranes in rats after cortical impact ${ }^{32)}$ and attenuate the inflammatory response in rat after brain injury. ${ }^{33)}$ HXY-059 (2,4-disulfonyl $\alpha$-phenyl tertiary butyl nitrone, now called HPN-07) has been used to reduce loss of injured brain tissue and increase cognitive function in rats with brain injury after percussion exposure. ${ }^{34)}$

The pharmacological interventions to block or inhibit some of the auditory pathological consequences caused by blast exposure have also been developed with antioxidant drugs (HPN07 and NAC). HPN-07 has been administered to prevent or treat acute acoustic trauma due to its primary ability to trap free radicals and its antioxidant properties, its action on important membrane enzymes (ion transport proteins), its role as a neuroprotectant, and its action as anti-inflammatory agent. ${ }^{2,3,35)}$ NAC has also been used to prevent or treat acute acoustic trauma due to its action as a ROS scavenger, its ability to reduce mitochondrial injury, its function to inhibit glutamate excitotoxicity, inflammation and apoptosis, and its role as neuroprotectant by improving levels of glutathione in the process of oxidative stress. ${ }^{2,3,10,28,30,35)}$ A combination of antioxidant drugs (HPN-07 and NAC) has been administered to reduce blast induced cochlear damage and hearing loss. ${ }^{10)}$ The combination of two antioxidant drugs (HPN-07 and NAC) showed a significant reduction in auditory brainstem response threshold shifts and distortion product otoacoustic emission level shifts at 2-16 kHz with significant reduction in inner hair cell and outer hair cell loss after blast exposure. ${ }^{10)}$ These results provide the first evidence that the combination of the antioxidant drugs can both enhance the recovery of temporary hearing loss and prevent permanent hearing loss by reducing damage to the mechanical and neural component of the auditory system. This study were specific and valuable because of minimizing or eliminating the possible sources of high variability among animals by placing the rat in a restraining tube that shielded its torso from the blast overpressure and by excluding ears with ruptured tympanic membranes from the study.

Clinically relevant and good animal models for studying the mechanisms and treatment of blast induced hearing loss should meet the following criteria: 1) the blast should be clearly identified and reproduced in controlled, reproducible, and quantifiable manner, 2) the injuries such as hearing threshold shift of auditory brainstem response, distortion product otoacoustic emissions, and inner and outer hair cells should be reproducible, quantifiable, and mimic the components of human blast induced neurotrauma, 3) the injury outcomes established based on the morphological, physiological, biochemical, and/or behavioral parameters should be related to the conditions of the blast, and 4) the mechanical properties (intensity and duration) of the blast should predict the outcome severity. $^{36)}$

\section{Conclusion}

Although many literatures related with blast induced hearing loss have been reviewed in this study, researchers are progressively developing the understanding of the vital mechanisms underlying blast induced hearing loss or blast induced injury. Because the current knowledge of the effects of blast on the auditory systems is still limited, controversial, and conflicting, there is an overt and strong need for clear and comprehensive understanding of the underlying mechanisms of the blast induced hearing loss. This can lead to a discovery of pharmacological treatment of the blast induced hearing loss. Finding a pharmacological intervention for blast related injury can only be established through a well-coordinated, multidisciplinary, and challenging research team consisting of physicists, pharmacologists, military scientists, biomedical researchers, audiologists, and other clinicians. Particularly, considering that all Korean men experience blast exposure through mandatory military service, research of the blast overpressure on human should be widely achieved in near future.

\section{Acknowledgments}

This study was supported by the Catholic University of Daegu and Research Institute of Biomimetic Sensory Control. 


\section{REFERENCES}

1) Choi $\mathrm{CH}$. Mechanisms of Noise-Induced Hearing Loss and Treatment. Korean Acad Audiol 2011;7:124-32.

2) Choi $\mathrm{CH}$. Preliminary study of the therapeutic effect of a nitronebased antioxidant drug (HPN-07) on acute acoustic trauma. Korean J Commun Disord 2011;16:202-10.

3) Choi $\mathrm{CH}$. Therapeutic effect of combined antioxidant drugs (4OHPBN plus NAC) on acute acoustic trauma in terms of distortion product otoacoustic emission. Korean Acad Audiol 2011;7:51-9.

4) Rovig GW, Bohnker BK, Page JC. Hearing health risk in a population of aircraft carrier flight deck personnel. Mil Med 2004;169:42932.

5) Garth RJ. Blast injury of the auditory system: a review of the mechanisms and pathology. J Laryngol Otol 1994;108:925-9.

6) Garth RJ. Blast injury of the ear: an overview and guide to management. Injury 1995;26:363-6.

7) Cave KM, Cornish EM, Chandler DW. Blast injury of the ear: clinical update from the global war on terror. Mil Med 2007;172:726-30.

8) Patterson JH Jr, Hamernik RP. Blast overpressure induced structural and functional changes in the auditory system. Toxicology 1997; 121:29-40.

9) Argyros GJ. Management of primary blast injury. Toxicology 1997; 121:105-15.

10) Ewert DL, Lu J, Li W, Du X, Floyd R, Kopke R. Antioxidant treatment reduces blast-induced cochlear damage and hearing loss. Hear Res 2012;285:29-39.

11) Hoffer ME, Balaban C. Neurotologic consequences of blast injury. International Brain Injury Association. NeuroTrauma Letter 05 2012 [cited 2012 Oct 15]. Available from: URL: http://www.internationalbrain.org/?q=node $/ 151$.

12) Elder GA, Cristian A. Blast-related mild traumatic brain injury: mechanisms of injury and impact on clinical care. Mt Sinai J Med 2009; 76:111-8.

13) Saïssy JM. Blast injuries. Curr Anaesth Crit Care 1998;9:58-65.

14) Mayorga MA. The pathology of primary blast overpressure injury. Toxicology 1997;121:17-28.

15) Kumar M. Blast injuries. Med J Armed Forces India 2010;66:309-11.

16) Harrell R. Pure tone evaluation. In: Katz J. Handbook of Clinical Audiology, 5th ed. Baltimore: Lippincott Williams \& Wilkins;2002. p.71-87.

17) Pahor AL. The ENT problems following the Birmingham bombings. J Laryngol Otol 1981;95:399-406.

18) Kronenberg J, Ben-Shoshan J, Modan M, Leventon G. Blast injury and cholesteatoma. Am J Otol 1988;9:127-30.

19) Roberto M, Hamernik RP, Turrentine GA. Damage of the auditory system associated with acute blast trauma. Ann Otol Rhinol Laryngol Suppl 1989;140:23-34.

20) Yokoi H, Yanagita N. Blast injury to sensory hairs: a study in the guinea pig using scanning electron microscopy. Arch Otorhinolar- yngol 1984;240:263-70.

21) Sudderth ME. Tympanoplasty in blast-induced perforation. Arch Otolaryngol 1974;99:157-9.

22) Singh D, Ahluwalia KJ. Blast injuries of the ear. J Laryngol Otol 1968;82:1017-28.

23) Mao JC, Pace E, Pierozynski P, Kou Z, Shen Y, VandeVord P, et al. Blast-induced tinnitus and hearing loss in rats: behavioral and imaging assays. J Neurotrauma 2012;29:430-44.

24) Kocsis JD, Tessler A. Pathology of blast-related brain injury. J Rehabil Res Dev 2009;46:667-72.

25) Säljö A, Bao F, Haglid KG, Hansson HA. Blast exposure causes redistribution of phosphorylated neurofilament subunits in neurons of the adult rat brain. J Neurotrauma 2000;17:719-26.

26) Yilmaz $S$, Pekdemir M. An unusual primary blast injury Traumatic brain injury due to primary blast injury. Am J Emerg Med 2007;25: 97-8.

27) Evans P, Halliwell B. Free radicals and hearing. Cause, consequence, and criteria. Ann N Y Acad Sci 1999;884:19-40.

28) Choi CH, Chen K, Vasquez-Weldon A, Jackson RL, Floyd RA, Kopke RD. Effectiveness of 4-hydroxy phenyl N-tert-butylnitrone (4-OHPBN) alone and in combination with other antioxidant drugs in the treatment of acute acoustic trauma in chinchilla. Free Radic Biol Med 2008;44:1772-84

29) Choi CH, Chen K, Du X, Floyd RA, Kopke RD. Effects of delayed and extended antioxidant treatment on acute acoustic trauma. Free Radic Res 2011;45:1162-72.

30) Du X, Chen K, Choi CH, Li W, Cheng W, Stewart C, et al. Selective degeneration of synapses in the dorsal cochlear nucleus of chinchilla following acoustic trauma and effects of antioxidant treatment. Hear Res 2012;283:1-13.

31) Chavko M, Adeeb S, Ahlers ST, McCarron RM. Attenuation of pulmonary inflammation after exposure to blast overpressure by $\mathrm{N}$-acetylcysteine amide. Shock 2009;32:325-31.

32) Xiong Y, Peterson PL, Lee CP. Effect of N-acetylcysteine on mitochondrial function following traumatic brain injury in rats. J Neurotrauma 1999;16:1067-82.

33) Chen G, Shi J, Hu Z, Hang C. Inhibitory effect on cerebral inflammatory response following traumatic brain injury in rats: a potential neuroprotective mechanism of $\mathrm{N}$-acetylcysteine. Mediators Inflamm 2008;2008:716458.

34) Clausen F, Marklund N, Lewén A, Hillered L. The nitrone free radical scavenger NXY-059 is neuroprotective when administered after traumatic brain injury in the rat. J Neurotrauma 2008;25:1449-57.

35) Floyd RA, Kopke RD, Choi CH, Foster SB, Doblas S, Towner RA. Nitrones as therapeutics. Free Radic Biol Med 2008;45:1361-74.

36) Cernak I, Noble-Haeusslein LJ. Traumatic brain injury: an overview of pathobiology with emphasis on military populations. J Cereb Blood Flow Metab 2010;30:255-66. 